\title{
THE ECONOMICS OF SOFTWARE 2.0
}

\author{
Felician ALECU \\ Bucharest University of Economic Studies, Romania \\ felician.alecu@ie.ase.ro \\ Lorena BĂTĂGAN \\ Bucharest University of Economic Studies, Romania \\ lorena.batagan@ie.ase.ro \\ Răzvan DINA \\ Bucharest University of Economic Studies, Romania \\ rdina@ase.ro
}

\begin{abstract}
Software 2.0 is completely focused on the expected behavior of a program, writing an application being about training a neural network rather than writing the code line by line. The programmers of tomorrow will most probably be data scientists rather than code writers. Artificial intelligence will very soon be accountable for the execution of the most of our repetitive tasks, while the humans will use their creativity for complex assignments for a greater economic value and an enhanced level of productivity. The first place in any ranking related to the artificial intelligence frameworks and libraries is occupied by TensorFlow. From an economic point of view, TensorFlow is one of the best choices for companies and individuals, having a significant contribution for the better world of tomorrow.
\end{abstract}

Keywords: Software 2.0, artificial intelligence, software development, automation, job markets, TensorFlow.

JEL classification: L86, C88, Y80

DOI: $10.12948 / \mathrm{ie} 2019.06 .09$

\section{Introduction}

Today, most of the software we use is manually created by programmers that are writing explicit instructions for the computers with the help of traditional programming languages like C, Java or Python and powerful IDEs.

Traditional programming is about instructing the computer line by line. In order to obtain a program that works in a satisfactory fashion, the programmer should take into account all the possible situations and particular circumstances the program may face during its execution, otherwise the program output will be an erroneous one. The situation becomes even more complicated when teams of developers are writing parts of the code intended to be finally assembled together since issues related to proper communication and integration between these modules are very common.

Software 2.0 is completely changing this classical model of programming by moving the focus on the desired behavior of the program rather than on describing all the steps needed for the program to achieve a specific goal [1].

When we speak about Software 2.0, we actually discuss about a combination between deep learning, neural networks and artificial intelligence. The programmer is not anymore writing the program explicitly, he only needs to design the skeleton of the program in terms of neural network architecture and data to be used to feed the system, while the program is doing the job to fulfil the desired behavior set by the developer.

Software 2.0 is not the end of the programmers even if the first thing we have in mind when we speak about Software 2.0 is the concept of software that is automatically writing itself. 
Working on the data the neural network is actually using will be an important activity of the programmers of the future because the quality of the results directly depends on the quality of the data the system is fed with. So, the future programmers will very likely be a sort of data scientists preparing the right data for the artificial intelligence programs and helping the teachable machines to reach an accuracy rate very close to $100 \%$.

\section{The twilight of Moore's Law}

The advances in information technology have been fundamental for the economic growth at the global level. We speak about an exponential grow as it has been stated by Gordon Moore, the Intel company cofounder, around 50 years ago, that noticed the computer processing power doubles every 1.5 years [13]. The economic implications are quite deep, we speak not only about an exponentially increased computer performance, but we also notice the costs of running the computers are continuously decreasing at an exponential scale too. In other words, if we wait, tomorrow we can by a better computer than today at a lower price. Surprisingly enough, Moore's Law prediction has been accurate for more than a half of a century.

Today we are facing a turning point regarding the Moore's Law since the exponential advances in terms of hardware are quite slower than the exponential technological demand, so we cannot relay exclusively on the Moore's prediction anymore in terms of the overall economic growth. In the shadow of the Moore's Laws twilight, some new opportunities are emerging, like Software 2.0, an environment in which making a computer program is mainly about training a neural network than writing the lines of code.

\section{Software 2.0 - advantages and (some) disadvantages}

What makes Software 2.0 so attractive for the people and companies? First, designing a neural network is a much easier activity compared with the writing of the code for a classical program, mainly being about matrix multiplications and activation functions, so we speak about a very limited set of instructions that makes neural networks to properly work even on devices with very low hardware capabilities like the development boards.

Since we are mainly speaking about activation functions and matrix multiplications, so a very limited set of options, portability is not a problem at all. Also, by adding or removing nodes, the program can be properly tuned in terms of performance and demanded resources.

Predictability is another great feature of neural networks since each iteration is involving the same amount of processing work and the same quantity of memory needed by the program to finish its execution.

About the limitations, the main disadvantage is related to the accuracy level that is lower than $100 \%$, so a low confidence level about the results may be encountered for some critical applications. The decision about trusting a 99\% accuracy level depends by the context, it could be very reasonable for the weather prediction but totally inappropriate for decisions involving questions on life and death.

\section{AI and the job market - the good and the bad}

Some voices are arguing about the fact artificial intelligence will first destroy our jobs and then the entire humanity. Other voices are simply saying the artificial intelligence will actually save us on the long run.

As any human advance, it's very important who and how is using the artificial intelligence, so we may presume the good will win, as always, so the artificial intelligence will have a very positive influence over the overall human life since the technology simply cannot completely replace the human mind mainly because in the end it is not a human-like product.

The positive influence of the Software 2.0 is related to the new jobs that will appear in the field 
of data engineers and data scientists - as we said before, the programmer of tomorrow will most probably be a data scientist rather than a code writer. Programmers should better teach the machines to become more human by deeply understanding not only the human speech but also the feelings and emotions.

On the other hand, jobs with a great automation potential are the most exposed to be replaced by the artificial intelligence, like the retail, transportation, manufacturing, constructions, health sector, education and so on. And all the other jobs will be affected by these substantial changes involved by the automation.

We do not see yet a computer like a danger, but the perception is totally different when we speak about robots and other computer-like devices powered by artificial intelligence. Some workers are already fear about the fact the near future will leave them out of a job, a situation very similar with the concerns the society faced during the industrial revolution. But, like it happened before during historical times, the change could be very useful for us, since we can delegate all our repetitive tasks (actions requiring attention and not intelligence, exposed to common mistakes and errors) to computers, being able to focus our human creativity on actions requiring a higher level of complexity.

The companies of tomorrow will divide the work to be done in two main categories, depending on the routine level of the task, so the automation can deal with the repetitive actions while the tasks for the humans will involve creativity, automation being used to empower the humans' capabilities, not to replace them. As illustrated in Figure 1, there are three main automation opportunities for the everyday work, each of these options having a positive influence over the human productivity and results.

\section{Enablers of work automation}

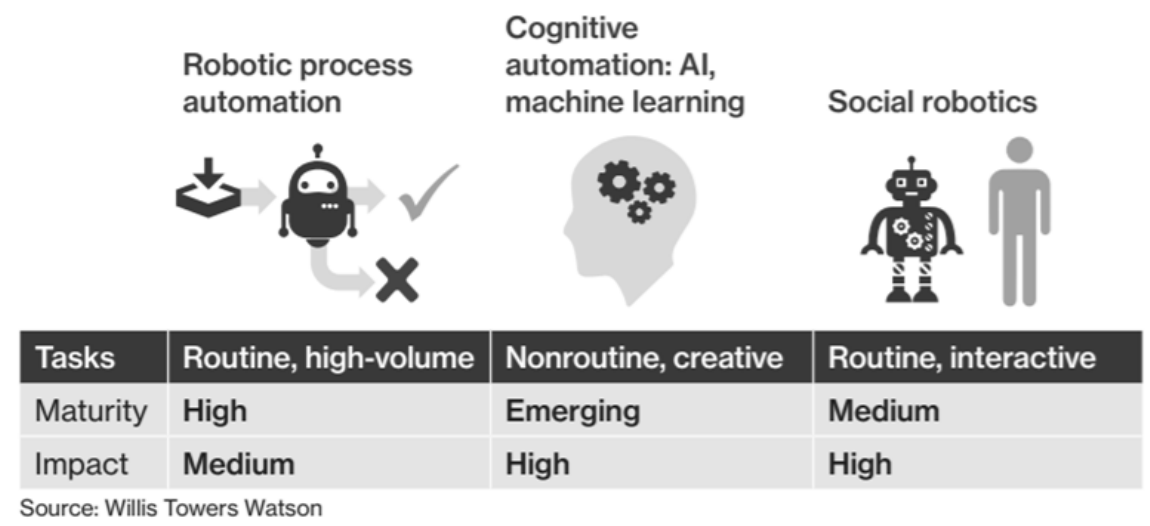

Figure 1. Automation opportunities [2]

The most mature one is the robotic process automation (RPA), aiming the high volume and low complexity routine tasks in any business organization, like dashboards for managers presenting statistics and progress that are automatically computed based on the people input in the work tracking system. The impact is at a medium level and there are no significant threats related to labor substitution.

Cognitive automation is about artificial intelligence empowering people to take better business decisions by augmenting the human capabilities.

Finally, the social robotics is about the real-life interactions between humans and machines, like robots making hamburgers or pizza in front of the customers that are enjoying this exceptional experience.

We can say the artificial intelligence will actually make humans more valuable since the technology appetite for quality accurate human interpretations is growing at a higher rate, so 
new unanticipated options will arise for the humans to add a greater economic value by augmenting our skills of today. The immediate result of such collaboration between humans and artificial intelligence artifacts is an enhanced level of productivity.

\section{Artificial Intelligence Frameworks and Libraries}

Since artificial intelligence and neural networks are the future of programming, some of the most advanced tools, frameworks, languages or libraries a developer may use today in this context of Software 2.0 are the following [3]: TensorFlow, Torch, Caffe, Theano, Amazon Machine Learning, Accord.Net, Scikit-learn, Apache Mahout, Microsoft CNTK (Cognitive Toolkit), Keras etc.

TensorFlow occupies the first place in any ranking related to the artificial intelligence frameworks and libraries thanks to the following two main reasons - it is open source and it is already used by some of the most important companies of our days, like Google, Intel, AMD, SAP, etc. Also, it is well suited for any type of program, from the simplest ones to the highly complex applications.

In 2015, Google open-sourced its machine-learning technology under the name TensorFlow, this move making TensorFlow to quickly become the $1^{\text {st }}$ choice for programmers and developers in the field of neural networks and artificial intelligence. This strategy is very similar with what happened with Android few years ago, making it open-source and freely available to virtually anyone helped the operating system to constantly consolidate its market share on smartphones, the current figures showing the fact around $80 \%$ of the smartphones worldwide are now powered by Android [4].

The core library of TensorFlow is written in $\mathrm{C}++$ and it runs on various operating systems and architectures [5]. TensorFlow can be also used on mobile devices, where the memory size is the main constraint [6].

Specialists are claiming the TensorFlow is a few years ahead of its competition [7], being a very attractive open source platform for the new users, making it the preferred choice of many programmers.

\section{Applications of TensorFlow}

TensorFlow is an open-source platform for machine learning allowing the users to design, build and deploy machine learning models. For any application, TensorFlow uses dataflow graph to represents the required computations [5]. The most effective applications made with TensorFlow are in the areas of labeling pictures/videos, like [8], biometrics (face, finger or eye recognition), understanding the handwriting, lips reading, handwriting simulation, music generation, Shakespeare translation and so on. Any condition related to classifications based on sample data is very suitable to be addressed with TensorFlow library.

There are many applications in the field of economics, like modules intended for financial models, financial data analysis or components made to predict the future prices or the fuel efficiency.

Some big companies are already using the TensorFlow to maximize their economic outcome, as the following [9]:

- Airbnb - is using TensorFlow to label the images the customers are uploading about properties, so the application is able to recognize the types of the rooms and other specific objects inside or outside (like a pool) for a better end-user experience on the platform;

- Alibaba - to optimize the ordering flow and to provide users a better application experience;

- Airbus - is extracting relevant details from satellite images by the use of TensorFlow;

- China Mobile - to detect and predict network anomalies and cutovers;

- Coca Cola - for the loyalty programs involving the scanning of the codes on the inner side 
of the caps by using the mobile phone camera;

- General Electric Healthcare - for a better classification of the magnetic resonance imaging;

- Google - is intensively sing TensorFlow in some of its well-known products, like Mail, Translate or Search;

- Intel - is using the TensorFlow capabilities to improve several product lines;

- Lenovo - is consolidating its Intelligent Computing Orchestration with the heavy help of TensorFlow;

- PayPal - for fraud detection based on fraud patterns;

- Qualcomm - making TensorFlow available on its platform and devices;

- Twitter - for ranking the tweets that appear on everyone's timeline;

\section{TensorFlow applications for non-experts}

Apart this big companies that are analyzing huge amounts of data for economic benefits, TensorFlow leaves a door open for real life domestic applications being able to quickly and easily generate economic value from data.

For example, a farmer from Japan named Makoto Koike managed to write his own TensorFlow application being able to sort cucumbers in 9 different classes based on size, shape, color, quality, freshness and so on [10]. Sorting the cucumbers is a complicated and time-consuming activity for humans, in the peak time of the crop one person being required to work on this type of job in a fulltime fashion.

By using the TensorFlow sample code and the tutorials, it was quite easy to quickly design his own application able to differentiate the cucumbers by simply analyzing their images taken by 3 cameras in three different ways, as illustrated in Figure 2.

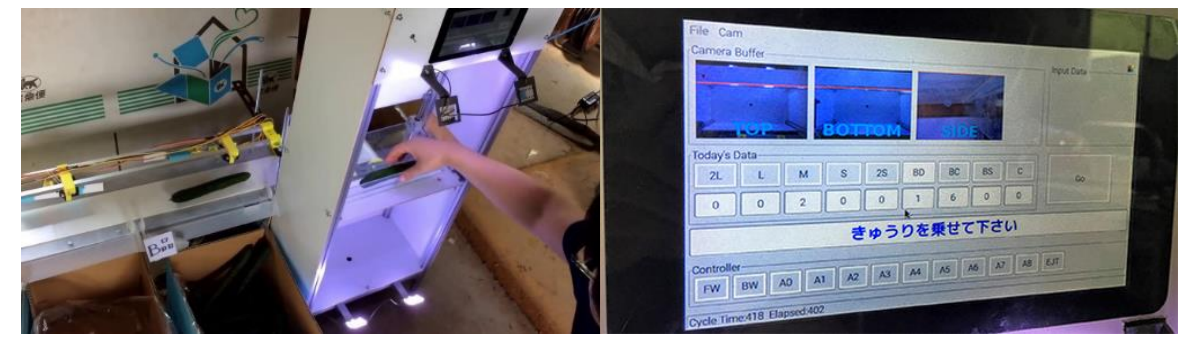

Figure 2. TensorFlow cucumber classification based on photo stand images taken with three cameras [12]

Makoto Koike said his idea was inspired by AlphaGo, a Google application that defeated the world champion of Go [11]. The AlphaGo victory is considered to be an important turning point for artificial intelligence because finally it was taken very seriously for the first time. So, as we expect to happen on a large scale in the future, our farmer was not terrified by the success of AlphaGo, so he tried to get some economical advantage on his own inspired by this great achievement of the artificial intelligence of our days. So, the technology is replicating the human capabilities in such a way the computer is able to perform this boring, repeating and time-consuming job of cucumber sorting while the human can use his knowledge and creativity to make a better farming.

Many non-experts are now discovering it is quite easy to write a TensorFlow application just starting from the examples, sample programs and tutorials, so a lot of pages in the Internet are posting interesting projects being able to recognize human generated inputs, like drawings, hand-writing, lip-reading, biometrics, etc.

From an economic point of view, for companies but also for individuals, TensorFlow is one of the best choices in terms of resources needed and results expected. TensorFlow still generates amazing results even if the training of the network is done with only a few sample data. 
www.conferenceie.ase.ro

\section{Conclusions}

In some fields, like classifications and object recognition, artificial intelligence is going very close to human level performance, and this situation will be better and better in the near future. We can clearly see how Google wants to dominate the artificial intelligence field by making it as simple and accessible as possible.

By democratizing the power of artificial intelligence and neural networks, TensorFlow made possible the appearance of domestic applications made by non-experts aimed to solve common economic issues, like the sorting of cucumbers in order to maximize the economic benefits coming from the crops.

This unique combination between human ingenuity and artificial intelligent is having great chances to make the world better.

\section{References}

[1] A. Karpathy, Software 2.0, Available at: https://medium.com/@karpathy/software-2-0a64152b37c35, Nov 11, 2017

[2] The future of work: Debunking myths and navigating new realities, Feb. 6, 2018, Available at: https://www.willistowerswatson.com/en/insights/2018/02/the-future-of-workdebunking-myths-and-navigating-new-realities

[3] A. Shaleynikov, Best Frameworks and Libraries for AI, Jan. 10, 18, Available at: https://dzone.com/articles/progressive-tools10-best-frameworks-and-libraries

[4] Smartphone Market Share, Available at: https://www.idc.com/promo/smartphone-marketshare/os

[5] M. Abadi et al., "TensorFlow: A system for large-scale machine learning," in Proc. of the 12th USENIX Symposium on Operating Systems Design and Implementation (OSDI 16), 2016, pp. 265-283

[6] Y. Chen, S. Biookaghazadeh, and M. Zhao, "Exploring the Capabilities of Mobile Devices Supporting Deep Learning," in Proc. of the 27th International Symposium on HighPerformance Parallel and Distributed Computing (HPDC '18). ACM, New York, NY, USA, pp. $17-18$

[7] Forbes, Reasons Why Google's Latest AI-TensorFlow is Open Sourced, Available at: https://www.forbes.com/sites/greatspeculations/2015/12/01/reasons-why-googles-latestai-tensorflow-is-open-sourced

[8] F. Eiler, S. Graf and W. Dorner, "Artificial intelligence and the automatic classification of historical photographs," in Proc. of the Sixth International Conference on Technological Ecosystems for Enhancing Multiculturality (TEEM'18), Francisco José García-Peñalvo (Ed.). ACM, New York, NY, USA, pp. 852-856

[9] Why TensorFlow?, Available at: https://www.tensorflow.org/about/case-studies/

[10] M. Helms, S. V. Ault, G. Mao, and J. Wang, "An Overview of Google Brain and Its Applications," in Proc. of the 2018 International Conference on Big Data and Education (ICBDE '18). ACM, New York, NY, USA, pp. 72-75.

[11] A. Zeeberg, "D.I.Y. Artificial Intelligence Comes to a Japanese Family Farm," The New Yorker, August 10, 2017, Available at: https://www.newyorker.com/tech/annals-oftechnology/diy-artificial-intelligence-comes-to-a-japanese-family-farm

[12] K. Sato, How a Japanese cucumber farmer is using deep learning and TensorFlow, Aug. 31, 2016, Available at: https://cloud.google.com/blog/products/gcp/how-a-japanesecucumber-farmer-is-using-deep-learning-and-tensorflow

[13] G. E. Moore, "Cramming more components onto integrated circuits," Electronics, Vol. 38, Number 8, Apr. 19, 1965, pp. pp.114-117 